\title{
XXI. Recent investigations bearing on the theory of electrolytic dissociation
}

\section{Louis Kahlenberg Ph.D.}

To cite this article: Louis Kahlenberg Ph.D. (1905) XXI. Recent investigations bearing on the theory of electrolytic dissociation, Philosophical Magazine Series 6, 9:50, 214-229, DOI: 10.1080/14786440509463273

To link to this article: http://dx.doi.org/10.1080/14786440509463273

册 Published online: 08 Jun 2010.

Submit your article to this journal $\lceil\pi$

Џ Article views: 2

Q View related articles $\square$

Citing articles: 1 View citing articles $\square$ 
people who have not yet surmounted all its difficulties nor forgotten the hard labour they went through in mastering the rudiments of it, should discuss just such points as are brought out so interestingly in the paper by Professor Orr which was the stimulus for the foregoing remarks.

Washington, D.C., U.S. Dept. of Agriculture, November $1,1904$.

XXI. Recent Investigations bearing on the Theory of Electrolytic Dissociation. By Lours Kahlenberg, Ph.D., Professor of Physical Chemistry in the University of Wisconsin*.

TN creating the theory of electrolytic dissociation the phe1 nomena of actual electrolysis have played a minor part. The changes of concentration of the electrolyte around the electrodes, the elevation of the temperature observed during the passage of the current, and the fact that when polarization is prevented, electrolytes obey Ohm's law, were all explained many years before the existence of the theory of electrolytic dissociation. Indeed, the hypothesis of Clausius, which displaced the old Grotthus theory and brought conceptions of electrolysis into harmony with thermodynamic requirements, is by many chemists and physicists still regarded as the best mechanical explanation of the electrolytic process, when all facts are fully considered.

Briefly stated, the theory of electrolytic dissociation is based upon the observation that for a goodly number of aqueous conducting solutions the so-called molecular conductivity increases with the dilution; that osmotic -pressure, lowering of the freezing-point, elevation of the boiling-point, or lowering of the vapour-tension of such solutions is abnormally great (i.e., that it is greater than for non-conducting solutions, the behaviour of which it is asserted conforms to the requirements of the gas equation); and that the so-called degree of electrolytic dissociation may be calculated from the electrical conductivity of the solution or from its osmotic pressure, its boiling-point, freezing-point. or vapour-tension. Indeed, the dissociation hypothesis is vitally connected with van't Hoff's theory of solution. The latter claims that dilute solutions behave according to the equation $\mathrm{PV}=i \mathrm{RT}$, in which the factor $i$ is unity for non-electrolytes. The analogy between a gas and a solution has been recognized so long that it would be difficult to state when the idea originated; but van't Hoff, by using Pfeffer's direct measurements of

* Communicated by the Faraday Society. Read before the Society, November 23, 1904. 
osmotic pressure, sought to show that dilute solutions obey the gas laws. In this he was unsucessful, in that he had to introduce the factor $i$ above mentioned, which varies for different solutes in the same solvent, and even for different concentrations of the same solute in a given solvent. An examination of the original tables published by Arrhenius* shows that the factor $i$ is by no means always unity for aqueous non-electrolytic solutions, nor does it always vary more from unity in the case of solutions of very considerable conductivity than in the case of non-electrolytes; but for ad goodly number of excellent electrolytes $i$ is materially greater than unity. The latter fact was utilized by Arrhenius in his endeavonr to show that the factor $i$ exceeds unity for electrolytic conductors, and increases with the dilution as does the so-called molecular conductivity. And thus upon the assumption that free ions, part-molecules charged with electricity, exist in an electrolyte and act like so many molecules, the general statement which van't Hoff' liad made for solutions was upheld-namely, that dilute solutions having the same temperature and the same osmotic pressure contain the same number of dissolved molecules.

Now I wish to contend that the facts presented by Arrhenius: in his original article are not sufficient to serve as a basis for the assumption of electrolytic dissociation in solutions; and that while isolated facts may here and there be interpreted by means of the dissociation theory, the bulk of experimental evidence gathered since 1887 speaks strongly against the theory.

In $1901+$ I published a list of results of cryoscopic and ebullioscopic determinations made with typical aqueous solutions of electrolytes and non-electrolytes, and also a list of molecular-conductivity determinations of the same electrolytes at $0^{\circ}$ and at $95^{\circ}$. It is unnecessary to discuss again the details of these results, which are rather voluminous. Suffice it to state that a comparison of the freezing-point values with the molecular conductivity at $0^{\circ}$, and also of the boiling-point values with the molecular conductivity at $95^{2}$, revealed the fact that there is no such connexion between freezing-points and boiling-points of solutions on the one hand, and their conductivity on the other, as is claimed by the theory of Arrhenius. In numerous cases not even a qualitative agreement exists. The facts presented in the paper cited have since been corroborated by Smits in his careful vapour-tension measurements, and by $\mathrm{H}$. C. Jones and co-workers in their

* Zeit. phys. Chem. i. p. 629 (1887).

+ Jour. phys. Chem. v. p. 339 (1901). 
molecular-weight determinations on solutions. The only objection which the adherents of the dissociation theory have made against using the data obtained as an argument against the dissociation hypothesis is that the solutions employed were not dilute enough, since the dissociation theory and the theory of van't Hoff hold only for infinitely dilute solutions, Now the work was performed with apparatus like that. used by Beckmann, and the lowest concentrations examined were such as could very well be tested by means of apparatus of that kind without incurring too large an experimental error ; $i . e$, the most dilute solutions tested were so chosen that the rise of the boiling-point or lowering of the freezing-point would as a rule be abont one-tenth of a degree. But stronger solutions, in some cases almost up to the point of saturation, were also examined in order to observe the changes of hoilingpoint and freezing-point with increase of concentration. The objection that all the solutions employed were too concentrated to enable one to use the data to confute the dissociation theory, may be met by the statement that the adherents of the theory, among them Arrhenius himself, have repeatedly used solutions as strong as and even stronger than the most dilute solutions I employed in the investigation cited ; for it is a fact known to all that the bulk of eryoscopic and ebullioscopic data gathered to support Arrhenius's claim of the relation between molecular conductivity and boiling-points and freezing-points have been obtained with the Beckmann apparatus, and hence the solutions used were as a rule not more dilute than those of lesser concentration employed by my co-workers and me. Not all the adherents of the theory of electrolytic dissociation would be ready to admit with Whetham* that the only satisfactory cryoscopic measurements at the extreme dilutions necessary to test the theory are those of Mr. E. H. Griffiths for cane-sugar and potassium chloride. It certainly is demonstrated by Griffiths's work that the so-called molecular lowering of the freezing-point of potassium chloride is within very small limits of experimental error double that of cane-sugar. This is merely one isolated experimental fact, and it has not yet been shown that the curve representing the change of the molecular conductivity with the concentration runs parallel with the curve indicating the change of the freezing-point with the concentration in the case of potassium chloride within the range investigated by Griffiths. Nor has this parallelism between freezing-point and molecular conductivity been rigidly demonstrated for the

* Paper read betore the Faraday Society, 'Electro-Chemist,' iii. July 1903, p. 16. 
solutions cryoscopically examined by Loomis. If one were therefore to take the attitude of Whetham, there would be no unobjectionable experimental evidence whatever on hand at present upon which to base the claim that freezing-points and molecular conductivity are related, as held by Arrhenius.

It may be asserted that all data of freezing-points and boiling-points of solutions on the one hand, and of molecular conductivities on the other, when carefully scrutinized, show that there is no such relation between them as the theory of electrolytic dissociation requires. While here and there isolated values of the degree of electrolytic dissociation, as calculated from molecular conductivities on the one hand and molecular-weight determinations on the other, agree approximately, this agreement is generally not within the limits of experimental error, and holds only for a very limited range of concentration.

In order to show that I was fully cognizant of the fact that the theories of van't Hoff and Arrhenius hold strictly only for infinitely dilute solutions, and that my argument against these hypotheses was based upon the general trend of the results, as varying from what would be expected were moderately concentrated solutions to behave like compressed gases, I should like to quote the following paragraph from my paper above cited :-

"I am well aware that the gas equation is supposed to hold strictly only for infinitely dilute solutions, just as it holds only for ideal gases, and that the solutions with which Dieterici worked varied between $0 \cdot 1$ and 1.0 normal, and that those used in experiments detailed above frequently were much stronger than normal. That a normal solution is nevertheless for many of the practical purposes of life a rather dilute solution will hardly be disputed. No one expects the gas equation to hold strictly for a normal solution or even for one considerably more dilute; but what one has a right to expect from the modern theory of solutions is, that with increasing concentration a solution should behave at least qualitatively as a gas does with increase of pressure. And this requirement is clearly not met, since while all gases behave alike under increase of pressure (so that van der Waals has been able to express their behaviour by means of his well-known equation) solutions, as has been shown, often behave in a manner opposite to that of gases, and this, too, frequently in solutions that cannot be termed concentrated. This demonstrates, then, that the van't Hoff law is at best only approximate and must be applied with great care. As Dieterici well says :-

" Raoult hat seine Gesetze der Dampfspannungs- und 
Gefrierpunkts-depression durchaus nicht als absolut streng gültige Naturgesetze aufgestellt, sondern als nahezu zutreffende Erfahrungssätze, welche für die Zwecke der Molekulargewichtsbestimmung genau genug sind." "

The molecular conductivity increases with the volume in very many cases; but in some instances, like that of the caustic alkalies, the molecular conductivity first increases and then decreases with increasing volume. In still other cases, the molecular conductivity diminishes or remains practically constant as the volume increases. The only cases which conform to the requirements of the dissociation theory, even in general trend, are those in which the molecular conductivity increases with the volume. Specific illustrations are given in the article cited above. The molecular conductivity at any volume $v$ is expressed by the equation $\Lambda_{v}=\mathrm{C} \times \mathrm{V}$, where $\mathrm{C}$ is the specific conductance of the solution, i. e. the reciprocal of the resistance of a cube of the solution having an edge of one centimetre, and $V$ is the volume in which one grammemolecule is contained. Now, as the specific conductance of a solution always diminishes as the solution is diluted, it is clear that in order that, $\Lambda_{v}$ may increase as the solution is diluted, the increase of the factor $\mathrm{V}$ must outweigh the diminution of $\mathrm{C}$. If $\mathrm{C}$ diminishes in the same ratio in which $\mathrm{V}$ increases, $\Lambda_{v}$ remains constant; and if (' diminishes more rapidly than $\mathrm{V}$ increases, $\Lambda_{v}$ will decrease as the volume becomes greater. As already stated, examples of all three cases are well known in practice.

It has been contended that it is the high specitic inductive capacity of the solvent that causes electrolytic dissociation. To demonstrate this, there are usually cited a few cases like benzene, chloroform, alcohol, and water, in which solvents, to be sure, the conductivity of solutions increases as the dielectric constant increases. However, in a series of articles published in the 'Journal of Physical Chemistry,' Schlundt and I have pointed out a goodly number of cases where solutions in solvents of low dielectric constant are better conductors than solutions in solvents with higher dielectric constants. Similar instances are constantly multiplying, now that methods of measuring specific inductive capacities have been simplifed and perfected. In the face of the fact that numerous exceptions to the Nernst-Thomson rule are known, and that no quantitative relation between the couductivity of a solution and the dielectric constant of its solvent has ever been established, it would appear that the Nernst-Thomson rule is really untenable.

Liquid hydrocyanic acid has a dielectric constant of about 
95, whereas the constant for water is about 80 . It is known that strong acids conduct exceedingly well in aqueous solution, but it has been shown that in liquid hydrocyanic acid they are very poor conductors*. In general, salts conduct much better in water than in liquid hydrocyanic acid. There are some exceptions to this, however; so, for instance, the salts of potassium tested conduct better in liquid hydrocyanic acid than in water. We have here a striking illustration of what is found continually, namely, that whether a solution will conduct electrolytically or not depends upon the specific character of both solute and solvent, and is quite independent of the dielectric constant of the solvent.

The approximately additive properties which some solutions exhibit has frequently been regarded as evidence in favour of the dissociation theory. Additive properties, however, are known to occur where electrolytic dissociation is out of the question, and hence these properties cannot be used as an argument in favour of the Arrhenius hypothesis. In the case of solids, the molecular heat is approximately equal to the sum of the atomic heats; in liquids, insulators as well as electrolytes, the molecular volumes and molecular refractions are approximately equal to the sum of the atomic volumes and atomic retractions respectively, and yet who would assume dissociation in these cases on that account?

If the dissociation theory be held, all the properties of a solution-pbysical, chemical, and physiological-must be ascribed to the properties of the ions and the undissociated molecules; and if the dissociation be assumed as nearly complete, the properties exhibited by such solution are to be ascribed largely to the properties of the ions. So the colour of aqueous electrolytes has been ascribed to the colour of the ions. Copper ions are blue; nickel ions are green; cobalt ions are red, \&c. But I have shown that benzene solutions of copper oleate, nickel oleate, and cobalt oleate are also blue, green, and red respectively, and that these solutions are as good insulators as benzene itselft. Furthermore, the cobalt-oleate solution turns blue when heated, and on cooling turns red again, exactly as aqueous conducting solutions of cobaltous salts. It has, therefore, been demonstrated that the colour of solutions is independent of their power to conduct the current, and cannot be used as an argument in favour of the dissociation hypothesis.

The reactions in aqueous solutions so commonly used in

* Compare Kahlenberg and Schlundt, Jour. phys. Chem. vi. p. 447 (1902)

+ Jour. phys. Chem. vi. p. 1. (1902). 
chemical analysis have been regarded as taking place between the ions in the solations, and the fact that these reactions proceed practically instantaneously in most cases, has beon ascribed to the peculiar electric properties which the ions are supposed to possess. But I have shown* that when copper oleate is dissolved in benzene and treated with $\mathrm{HCl}, \mathrm{PCl}_{3}$, $\mathrm{AsCl}_{3}, \mathrm{SnCl}_{4}$, or $\mathrm{SiCl}_{4}$, also dissolved in the same solvent, cupric chloride is precipitated in each case exactly as silver chloride is thrown down from an aqueous silver-nitrate solution by soluble chlorides. And furthermore, a slight excess of the precipitant in the case of the benzene solutions mentioned, yields more complete precipitation, just as it does in aqueous solutions. The benzene solutions mentioned are all excellent insulators. It is therefore demonstrated that the instantaneous reactions observed in aqueous electrolytic solutions cannot be ascribed to the fact that these solutions conduct electricity-or, in the language of the dissociation theory, to the presence of free ions-for exactly similar reactions occur in solutions that are the best of insulators. Such views, then, as are presented in Ostwald's 'Scientific Foundations of Analytical Chemistry,' and are echoed by various other writers, cannot be maintained.

According to the dissociation hypothesis, solutions of acids are supposed to owe their characteristic properties to the presence of hydrogen ions. Now it is well known that trichloracetic acid, when dissolved in water, yields an excellent electrolyte, and exhibits the properties of a fairly strong acid. In the course of an investigation $\dagger$ on the electrical conductivity of solutions in sulphocyanates and mustard-oils, I showed that a normal solution of trichloracetic acid in allyl mustard-oil is a poorer conductor than the purest water which Kohlrausch ever prepared in contact with air, and yet this solution attacks dry magnesium rapidly, and decomposes the dry carbonates of sodium and potassium. It is well known that even ordinary distilled water attacks magnesium quite slowly, and of course does not evolve carbon dioxide from alkaline carbonates. However, judging from the conductivity test, water contains more hydrogen ions than the solution of trichloracetic acid in allyl mustard-oil, and ought to show more vigorously the characteristic action of the hydrogen ion.

In physiological investigations, too, the theory of electrolytic dissociation has proved inadequate; so, for instance, it has been found that dilute solutions of acid salts are sourer

* Journ. phys. Chem. vi. p. 1 (1902).

+ Zeit. phys. Chem. xlvi. p. 64 (1903). 
in taste and more toxic towards plants than they ought to be according to their content of hydrogen ions, as determined by the methods in vogue*.

That the hypothesis of Arrhenius cannot be harmonized with the facts established in the domain of thermal chemistry has repeatedly been emphasized by various writers, and has been well summed up by Reychler in his treatise on physicochemical theories. The conclusion reached by Reychler is that "above everything else we notice that the hypothesis of free ions is in opposition to thermochemical observations."

It is a well-known fact that the dissociation theory cannot be harmonized with the law of mass action, and that this constitutes a strong argument against the hypothesis. For solutions of weak organic acids in water, to be sure, Ostwald succeeded in computing a series of constants by means of the formula expressing his well-known dilution law, growing out of an attempt to apply the law of mass action to electrolytic dissociation. But an examination of the constants obtained for these weak acids reveals the fact that the agreement is after all only rough ; furthermore, the attempt to apply the dilution formula to electrolytes par excellence has utterly failed, and yet the advocates of the dissociation theory continue to calculate the so-called solubility product, which frequently involves the application of the law of mass action to strong electrolytes. Attempts made by Lincoln to apply the dilution formula to electrolytes in non-aqueous solutions of about the same order of conductivity as that of some of the solutions of weak organic acids in water, have also proved fatile. How can the formula possibly hold in cases where the molecular conductivity remains practically constant, or decreases as the dilution increases? Rudolphi and also van't Hoff have sought to amend the formula by changing the exponents so that constants would result from the observed data in special cases. While thus better "constants" were obtained in these instances, the altered formula proved entirely unsatisfactory in other cases ; and then, too, it must be added that the alterations made the formulæ simply empirical in character, without any theoretical basis.

The difficulties met with in applying the Nernst formula for calculating the electromotive forces of galvanic cells bave been discussed in special papers to which the reader is referred $\uparrow$. It may be remarked here, however, that in the work on electromotive forces especially, many of the solutions

* Compare Jour. phys. Chem, ir. p. 33 (1900); iv. p. 207 ; iv. p. 533 ; iv. p. 553 .

† Joum. phys. Chem. iii. p. 379 (1899); also iv. p. 709 (1900). 
used by advocates of the dissociation theory were far from dilute; and, at best, the agreement between calculated and observed values leaves much to be desired.

Many colloidal solutions coagulate when treated with solutions that conduct electrolytically. Attempts have been made to ascribe this coagulative power to the free ions which the electrolyte is supposed to contain. It is true, however, that the power to coagulate colloidal solutions is by no means confined to electrolytic solutions; and hence any one claiming, for example, that sodium-chloride solution coagulates colloids because it is an electrolyte, must assume the burden of proving his proposition.

The theory of electrolytic dissociation is unable to furnish a satisfactory explanation of the phenomena in aqueous solutions, but it is in the realm of non-aqueous solutions that the hypothesis proves especially impotent. When the theory was promulgated but few non-aqueous solations had been examined as to their power to conduct the current, and those few solutions, in which ether, alcohol, chloroform, and hydrocarbons, for instance, were the solvents employed, were found to be poor conductors. So the idea that only aqueous solutions conduct electricity to any considerable extent gained ground and held sway for a number of years with remarkable tenacity, the view being strengthened by the fact that in the solvents just mentioned molecular weights as determined by boilingpoint, or, where possible, by freezing-point methods, were not "abnormally" low, as in the case of solutions of many ordinary salts in water. But now we know of non-aqueous solutions which are as good and even better conductors of electricity than aqueous solutions ; and these solutions, when tested by the methods of determining molecular weights just mentioned, yield results that are generally higher then those computed from the formulæ of the solutes; only exceptionally is the reverse the case. In the course of my work on nonaqueous solutions, I have pointed out numerous examples of this; and recently the splendid researches of Walden have established many more. His work on solutions in liquid sulpbur dioxide especially shows that in this solvent "abnormally" high molecular weights are generally found in the case of solutions of excellent conducting power. The field of non-aqueous solutions is a very large one, and it is here that the theory of electrolytic dissociation can really best be tested. It is here that the fact that the theory is untenable becomes particularly apparent. Whetham's statement* that work with non-aqueous solutions is rendered difficult by the * L. c. p. 19. 
fact that with some solvents like alcohol freezing-point work is impossible, is hardly valid, for such solutions may be readily examined by vapour-tension or boiling-point methods. It is true that in operating with many non-aqueous solutions, like liquid ammonia, liquid sulphur dioxide, liquid hydrocyanic acid, special difficulties and dangers present themselves; but these are generally quite surmountable, and indeed often it is fully as easy to investigate a non-aqueous solution as one in which water is the solvent. It simply has not yet become generally fashionable to investigate non-aqueous electrolytic solutions. When this work is taken in hand by more investigators, the fact that the dissociation theory is useless and misleading will gain ground more rapidly.

It has been argued by some that since the dissociation theory has been developed by use of aqueous solutions, the fact that it cannot explain the phenomena in non-aqueous solutions should not be used against it. It is well known, however, that defenders of the ionic hypothesis have continually sought to support their views by citing some phases of the behaviour of non-aqueous solutions. Thus in trying to find facts to support the Nernst-Thomson rule, in comparing chemical activity in electrolytes and non-electrolytes and in considering molecular weights in conducting and nonconducting solutions, the behaviour of non-aqueous solutions has always been compared with that of aqueous solutions. By so doing it has been virtually admitted that it is legitimate to apply the dissociation theory to non-aqueous solutions. It is only since the discovery of excellent electrolytes in solvents other than water, the behaviour of which constitutes a strong argument against the theory, that objections have been made to testing the theory in the field of non-aqueous solutions.

But the dissociation hypothesis has been applied even to electrolytes that are not solutions at all; to fused salts, for instance, to pure solvents, and to gases. The line of thought in this procedure is that, since the electrolytic conductivity of solutions is assumed to be due to free ions, free ions must occur wherever electrolytic conduction is found. And so we find the idea established that pure substances dissociate themselves electrolytically without the aid of any solvent whatever. Now since a pure substance may be considered as a solution 100 per cent. strong, it might be well for the advocates of the dissociation theory to pause to consider whether such solutions are sufficiently dilute to warrant the attempt to apply to them a theory which it is admitted holds strictly only for infinitely dilute solutions. Even conservative adrocates 
of the theory of electrolytic dissociation who question whether there is electrolytic dissociation in the case of fused salts, are ready to consider that the fact that the determination of the so-called degree of electrolytic dissociation of pure water by conductivity and electromotive-force measurements leads to approximately the same result, constitutes an argument in favour of the theory in question.

The theory of electrolytic dissociation and the van't Hoff theory of solutions have done much toward stimulating inquiry; but the discovery of many new facts has made them entirely untenable. I do not share the opinion that these hypotheses may be retained in a somewhat modified form. The observed facts that speak against them are by far too numerous; and furthermore, any theory of solution which holds strictly only for infinitely dilute solutions cannot be expected to maintain itself, for in reality it does not apply at all to any solutions with which we work in practice.

I have thus briefly rehearsed the reasons why the theories of Arrhenius and van't Hoff are untenable, to me a task far from agreeable, inasmuch as during my student days and for several years afterwards I was quite enthusiastic over these hypotheses, and it was while seeking to establish new experimental facts to further support the theories that contradictory phenomena were continually observed.

I should not like to close these pages without indicating the direction which in my opinion further investigations on solutions must take. All treatises on physics and chemistry seek to draw a sharp distinction between the processes of solution and chemical action ; the former is commonly described as purely physical in character, the latter as more deep seated and as caused by a specific attraction termed chemical affinity. It is well known that some substances react chemically (using that term in its usual sense) with each other, and again others do not; and that the rate of interaction and the final condition of equilibrium reached are subject to influences of temperature especially, but also to pressure and concentration. The same may be stated of the process of solution. Some substances dissolve each other, others do not; and again, the process, like that of chemical action, is subject to influences of temperature, pressure, and concentration. Energy changes, such as thermal changes, electrical changes, expansions and contractions, and evolutions of light, are known to accompany the processes of solution as well as those of chemical action. With the exception of the mass, the properties of chemical compound: are never exactly equal to the sum of the properties of the 
constituents entering into them ; the same is true of solutions. Furthermore, those properties which are approximately additive in character in the case of chemical compounds are also approximately additive in the case of solutions. Examples of such properties are the molecular volume, molecular refractive power, and molecular heat. Again, other properties like, for example, the optical rotatory power, the specific inductive capacity, which are not additive in chemical compounds, are also not additive in the case of solutions. In fact, the only distinction between a solution and a chemical compound is that the latter conforms to the laws of definite and multiple proportions and the former does not. But it must be borne in mind that somcalled pure chemical compounds are obtained only by subjecting the total reaction products to certain purifying processes, such as evaporation, distillation, sublimation, crystallization, washing, extraction, dc. All of these processes put the reaction products, which are in general solutions (using that word in its broader sense) under special duress, as a result of which there are obtained as cleavage pieces of these solutions, as it were, phases whose composition remains constant through a greater or lesser range of temperature, pressure, and contact with other phases. The processes of solution and chemical action are then identical in character, and chemical compounds are merely the cleavage pieces of solutions placed under special stress or duress represented by the so-called purifying processes. The process of solution is thas the general case of the interaction of bodies, union resulting when the specific attraction, commonly called chemical affinity, between them is under the existing conditions sufficient to cause an interpenetration, a fusion or blending of their masses as it were. Furthermore, adhesion, absorption, adsorption, and imbibition are also dut to the same specific attraction which causes solution and chemical action. Adhesion is really to be regarded as an unsuccessful attempt at solution. Thomas Graham was unquestionably right when he stated that from adhesion to solution and chemical action there is every stage of gradation. It is scarcely necessary to add that these views were also entertained by numerous other scientists of note, among whom Bunsen was especially prominent. The truth of this view will force itself upon the mind of any one who seeks to work out the problem of solutions in the laboratory rather: than at the writing-desk. It needs hardly to be emphasized that the recognition of the importance of the laws of definite and multiple proportions, which have been found to hold in so many cases of phases that maintain their composition for Phil. Mag. S. 6. Vol. 9. No. 50. Feb. 1905. 
considerable ranges of alterations of temperature, jressure, and nature of co-existing phases, is not at all affected by the above considerations. The discovery of these laws has been of inestimable value in analytical and synthetic work and in systematizing our knowledge; but it is a mistake to think that they necessitate the conclusion that the processes of solution and chemical action are different in character and are caused by different agencies. The fact that the quantities of similar solutes which must be added to equal quantities of a given solvent to produce solutions of the same vapour-tension, are to each other as the quantities in which such solutes unite with other substances to form stereotyped chemical compounds, constitutes a strong argument for regarding the processes of solution and chemical action as identical in character.

It thus becomes evident that in investigating solutions we must begin with the most concentrated and end with the most dilute; the latter will appear simply as a limiting case. So, for instance, the change of the vapour-tension of solutions with the concentration must be studied from the strongest solutions obtainable to the dilutest that can still be measured throughout the range of temperatures at which the solutions can exist at all. And this work must be done for a large number of solutes in a large number of solvents. Such data being at hand, the equations expressing the changes of vapourtension with temperature and concentration may be written. Work of this character has only barely begun; but judging from the results at hand, analogous substances will exhibit similar behaviour; and though it is not to be expected that one equation will serve for all solutions, similarities between the equations holding for different solutions will not be lacking.

According to the views here advanced-or, I had better say, revived - the molecular weights of, for instance, sodium and potassium salts in aqueous solutions as determined by the diminution of the vapour-tension, elevation of the boilingpoint, and lowering of the freezing-point, are not abnormally low because these salts are dissociated, but rather because they have great affinity for water. On the other hand, these salts have less affinity for liquid sulphur dioxide, as is indicated by the relatively high vapour-tension of the solutions and the correspondingly high molecular weights, though the solutions conduct well nevertheless. As an example of the other extreme, the molecular weights of colloids in water are not enormous; the results come out high simply because the affinity between water and these colloids is relatively slight. Furthermore, it is also easy to see why colloids are 
readily coagulated by substances which lower the vapourtension greatly, and that their coagulating power stands in relation to their ability to lower the vapour-tension ; but this coagulating power has nothing to do with electrolytic conduction. From the vapour-tension of a solution no one can predict whether that solntion will conduct electricity or not, much less tell how well it will conduct. This statement is fully warranted by the facts as known at present.

Many salts are very good electrolytes when in the molten state; others are poor conductors, and still others are insulators. From the views just advanced, it is clear that it is perfectly correct to consider electrolytic solutions and fused electrolytes together ; for conducting solutions are electrolytes which are in a fused condition at ordinary temperatures. Faraday selected the experiment in which a current is passed through fused silver chloride between two silver electrodes as representing the simplest ease of electrolysis; for here there are but two chemical elements involved, and yet all the phenomena accompanying electrolysis are here exhibited. Who would question the correctness of this view? The theory of electrolytic dissociation has led Whetham to consider the case of fused electrolytes as entirely different from that of electrolytic solutions, a position which is clearly not warranted by experimental facts.

Take water and pure acetic acid. Both are very poor conductors. Add a little water to some of the acid; and again add a little acid to some of the water. In both cases the resulting solutions conduct. Now is it the acid that conlucts in the water, or is it the water that conducts in the acid? Years ago it was customary to say that we acidulate the water to make it conduct better; now it is fashionable to say that it is the acid which in the dissociated state conducts in the water, the latter serving merely as a medium in which the ions of the acid may exist and migrate. Furthermore, the original conductivity of the pure water is frequently deducted from that of the solution, in order to determine the conductivity of the acid. As a matter of fact, however, it is the combination of the acetic acid and water that conducts. The water has just as much to do with the conduction as the acid, and it is absurd to subtract either the conductivity of the water or that of the acid from the conductivity of the solution. But the calculation of the molecular conductivity is founded upon the supposition that the solvent plays no part in the conduction. This supposition being untenable, it follows that the comparison of the molecular conductivities at different concentrations is a process which is quite Q 2 
unjustifiable. This becomes particularly apparent from the researches of Patten *, and recently C. J. Reed + has presented the subject in a very able manner before the American Electrochemical Society. The Kohlrausch law of the additive character of the molecular conductivity of solutions at very high dilutions holds at best only for a limited class of aqueous solutions: it is, of course, founded upon the calculations of molecular conductivity, which, it bas been pointed out, are objectionable. The whole matter of the calculation of so-called molecular conductivities of solutions at different concentrations being open to serious objection, it is not at all surprising that such a relation between electrical conductivity of solutions and their freezing-points or boiling-points as is claimed by the theory of electrolytic dissociation does not exist.

Faraday continually emphasized the view that in electrolysis the products do not appear at the electrodes because they are attracted there by the latter. His idea was that the products are ejected at the electrodes by the electrical energy acting as an axis of force driving the products out in the two directions, the electrodes serving simply as doors. In a special paper on electrochemical theories, to which the reader is referred $\ddagger$, I have entered somewhat more fully into a consideration of theories of electrolysis.

During the past year I have been engaged in osmotic experiments, which included both qualitative investigations and quantitative measurements of osmotic pressures. The latter determinations were made with semi-permenble membranes. The details of the apparatus used, and the results obtained, will soon be published in a special paper. Here I would like to state, however, that it was found that whether osmosis will take place or not depends on the nature of the membrane and the liquids bathing it; and the direction and magnitude of the osmotic pressure is also dependent upon the nature of the membrane and the liquids in contact with it. The quantitative measurements of osmotic pressure made with semipermeable membranes yielded results which are not such as the gas laws require. It is especially noteworthy that cases have been found in which at constant temperature the osmotic pressture diminishes very rapidly as the solution is diluted, becoming practically nil for solutions about one-twentieth normal. The results of the osmotic experiments are readily explained by the view that the membrane imbibes or unites with the solvent, because of the affinity existing between them,

* Journ. phys. Chem. vi. p. 554 (1904).

$\uparrow$ Trans. Amer. Electrochem. Soc. v. p. 108 (1904).

$\ddagger$ Trans. Amer. Flectrochem. Soc. i. p. 119 (1902). 
and then the solution extracts solvent from the membrane. The latter part of the process can only take place when the affinity of the solution for additional solvent exceeds that of the saturated membrane for solvent. It is clear that, as the solution is diluted, a strength will finally be reached where the affinity of the solution for additional solvent is just equal to that between membrane and solvent, and then osmosis will stop, i.e., the osmotic pressure will become nii. This eritical strength of the solution varies with the nature of the membrane and that of the solution; but in general it is far from infinitely dilute, being quite within the pale of what is readily measurable. The classical copper ferrocyanide membrane, being a colloid, has but slight affinity for water, which is readily extracted from it by a fairly dilute solution of sugar. Thus osmotic pressure so-called is due to the same affinity which causes adhesion, imbibition, absorption, adsorption, solntion, and chemical action.

Of late years work in physical chemistry has been largely directed to the study of the effects of temperature, pressure, and concentration on the progress of chemical reactions; and properly so. It has at times been forgotten, however, that affinity must exist before union can take place; and that temperature, pressure, and concentration are simply to be regarded as modifying factors aiding or retarding the tendency of affinity. By some it is even deemed as somewhat old-fashioned to talk about affinity. But upon the distinct recognition of affinity, and a careful investigation of the laws governing it, clearly depends the future progress of chemistry, physical chemistry, and physiology. We must learn to measure affinity quantitatively; but in order to take into account the phenomena of morphology as they confront us in crystals, but particularly in living beings, we need to learn to study the direction as well as the strength with which affinity acts under given conditions. Finally, the question why certain solutions, molten salts, \&c., conduct electricity and others do not, will probably not be answered until we can tell why a stick of silver conducts electricity and a stick of sulphur does not. These questions really involve a better understanding of the relation between electricity and gross matter, a problem which is apparently being attacked with promise by J. J. Thomson and his co-workers. Until we have more light on this subject, we can hardly hope for very material improvements of our views of the nature of electrolytic processes.

Laboratory of Physical Chemistry, University of Wisconsin, Madison, August, 1904. 